Schweizerisches Jahrbuch für Entwicklungspolitik

16 | 1997

Umwelt und Entwicklung, Rio : Fünf Jahre danach

\title{
Die Internationalen Verhandlungen Im Bereich Handel Und Umwelt
}

Sophie Forster

\section{OpenEdition}

1 Journals

Electronic version

URL: http://journals.openedition.org/sjep/749

DOI: 10.4000/sjep.749

ISSN: 1663-9677

Publisher

Institut de hautes études internationales et du développement

Printed version

Date of publication: 1 mars 1997

Number of pages: 211-221

ISSN: 1660-5926

\section{Electronic reference}

Sophie Forster, « Die Internationalen Verhandlungen Im Bereich Handel Und Umwelt », Schweizerisches Jahrbuch für Entwicklungspolitik [Online], 16| 1997, Online erschienen am: 13 August 2012, abgerufen am 08 September 2020. URL : http://journals.openedition.org/sjep/749 ; DOI : https://doi.org/ $10.4000 /$ sjep.749 


\title{
DIE INTERNATIONALEN VERHANDLUNGEN IM BEREICH HANDEL UND UMWELT
}

\author{
SOPHIE FORSTER*
}

\section{EINFÜHRUNG}

Seit dem berühmten « Thunfisch-Panel » des GATT im Jahr $1991^{1}$ bildet die Problematik Handel und Umwelt den Gegenstand zahlreicher Diskussionen in verschiedenen internationalen Gremien. Insbesondere nach der Konferenz der Vereinten Nationen über Umwelt und Entwicklung von 1992 und nach dem Abschluss der Uruguay-Runde im Jahr 1994 trat das Thema verstärkt in den Vordergrund.

Allgemein und sehr schematisch dargestellt werden die Diskussionen durch die Meinungsunterschiede zwischen Entwicklungs- und Industrieländern geprägt. Die Entwicklungsländer befürchten, dass Umweltpolitiken zu protektionistischen Zwecken benutzt werden (« grüner Protektionismus »).

Der vorliegende Artikel gibt einen Überblick über die Fortschritte, welche die wichtigsten internationalen Gremien, die sich mit der Beziehung zwischen Handel und Umwelt befassen, in den letzten Jahren erzielten. Im folgenden werden die Arbeiten der WTO, der UNCTAD und der OECD dargestellt. Da jedoch zur Zeit einzig die unter der Ägide der WTO durchgeführten Arbeiten in konkreten Massnahmen münden dürften (bei den übrigen Organisationen handelt es sich eher um Diskussions- und Analysestellen), werden diese eingehender behandelt. Im übrigen liegt der Schwerpunkt auf Themen, denen die Schweiz eine besondere Bedeutung beimisst, oder zu welchen bereits eine umfassende Diskussion stattfindet.

\section{STAND DER DISKUSSIONEN IN DER WELTHANDELSORGANISATION (WTO)}

\subsection{AUSSCHUSS FÜR HANDEL UND UMWELT}

Der Ausschuss für Handel und Umwelt (CTE) wurde im April 1994 durch den Ministerentscheid von Marrakesch als Nachfolger der Gruppe « Umweltmassnahmen und Welthandel $»^{2}$ eingesetzt. Der CTE erhielt das Mandat, die «Beziehung zwischen Handels- und Umweltmassnahmen » zu definieren, « um die nachhaltige Entwicklung zu fördern », und «geeignete Empfehlungen dazu » abzugeben, « ob die Bestimmungen des multilateralen Handelssystems gegebenenfalls zu ändern sind $»^{3}$. "Mit dem Ziel, sicherzustellen, dass die internatio-

\footnotetext{
* Die in diesem Artikel geüsserten persönlichen Meinungen sind lediglich für den Verfasser verbindlich.

1 Das Streitbeilegungsorgan des GATT befand, dass die USA mit dem Verbot von Thunfischimporten aus Mexiko gegen ihre internationalen Handelsverpflichtungen verstiessen. Das amerikanische Embargo war wegen der angewandten Fischmethoden verhängt worden, die den Tod zahlreicher Delphine verursachten.

2 Die Gruppe war zwar schon 1971 entstanden, wurde aber erst im Oktober 1991 auf die Initiative der Schweiz einberufen.

3 Entscheid über Handel und Umwelt vom 14. April 1994.
} 
nale Handelspolitik und Umweltpolitik einander gegenseitig fördern $»^{4}$, wurde ein relativ präzises Arbeitsprogramm verabschiedet (siehe Kasten).

Der CTE wurde beauftragt, auf der ersten Zweijahressitzung der Ministerkonferenz im Dezember 1996 in Singapur einen Bericht und Empfehlungen vorzulegen. Bislang wurden im CTE alle Punkte des Arbeitsprogrammes zwar besprochen, aber in unterschiedlichem Masse vertieft. In den nachstehenden Absätzen werden lediglich jene Themen zusammengefasst, welche echte Diskussionen auslösten.

\section{Arbeitsprogramm des CTE}

1. Verhältnis zwischen den Bestimmungen des multilateralen Handelssystems und den Handelsmassnahmen, die zum Zwecke des Umweltschutzes ergriffen werden, einschliesslich der Massnahmen aus multilateralen Umweltabkommen (MEA) ;

2. Verhältnis zwischen Umweltpolitiken, welche den Handel betreffen, Umweltmassnahmen mit erheblichen Auswirkungen auf den Handel und den Bestimmungen des multilateralen Handelssystems ;

3. Verhältnis zwischen den Bestimmungen des multilateralen Handelssystems und :

a) den aus Umweltgründen erhobenen Steuern und Gebühren,

b) den aus Umweltgründen vorgeschriebenen Auflagen zu Produkten, einschliesslich der technischen Normen und Regelungen sowie der Verpackungs-, Etikettierungs- und Recyclingvorschriften.

4. Bestimmungen des multilateralen Handelssystems zur Transparenz der aus Umweltgründen angewandten Handelsmassnahmen sowie zu Umweltmassnahmen und -vorschriften mit erheblichen Auswirkungen auf den Handel ;

5. Verhältnis zwischen den Streitbeilegungsmechanismen des multilateralen Handelssystems und jenen, die in den multilateralen Umweltabkommen vorgesehen sind ;

6. Auswirkungen von Umweltmassnahmen auf den Marktzugang für die Entwicklungsländer und insbesondere die am wenigsten entwickelten Länder; Umweltvorteile aus dem Abbau der Handelseinschränkungen und -verzerrungen ;

7. Frage der Ausfuhren von auf dem inländischen Markt verbotenen Produkten ;

8. Überprüfung der relevanten Bestimmungen des Abkommens über die handelsbezogenen Aspekte der Rechte an geistigem Eigentum ;

9. Überprüfung des im Beschluss zum Thema Dienstleistungsverkehr und Umwelt vorgesehenen Arbeitsprogramms ;

10. Beitrag zu geeigneten Vereinbarungen im Hinblick auf die Zusammenarbeit mit zwischenstaatlichen Organisationen und Nichtregierungsorganisationen laut Artikel V WTO.

4 Ibid. 


\subsection{MULTILATERALE UMWELTABKOMMEN (PUNKT 1 DES CTE-ARBEITSPROGRAMMS) UND STREITBEILEGUNG (PUNKT 5)}

\section{$\square$ Problematik}

Heute gibt es ungefähr 180 multilaterale Umweltabkommen oder -verträge. ${ }^{5}$ Die meisten davon wirken sich nicht auf den Handel aus. Nur rund zwanzig Abkommen enthalten Handelsmassnahmen, welche verschiedene Ziele verfolgen :

- Einschränkung des Handels zum Schutz der Umwelt oder zur Erhaltung natürlicher Ressourcen ;

- Anreiz für die Staaten, den Abkommen beizutreten (um das Phänomen der « free-riders », Trittbrettfahrer, zu vermeiden).

Handelsmassnahmen stehen nicht zwangsläufig im Widerspruch zu den Regeln der WTO. Im übrigen wurde offiziell noch keine handelspolitische Massnahme für mit den WTO-Disziplinen unvereinbar erklärt, da bislang auch keine Konflikte aufgetreten sind. ${ }^{6}$

Bedarf deshalb die Beziehung zwischen der WTO und den multilateralen Umweltabkommen (MEA) überhaupt einer Klärung ? Reicht Artikel XX des GATT, der unter bestimmten Voraussetzungen « Massnahmen, die für den Schutz des Lebens oder der Gesundheit von Pflanzen und Tieren oder die Erhaltung des Pflanzenwuchses erforderlich sind", und "Massnahmen, die zum Schutz natürlicher Hilfsquellen, bei denen die Gefahr der Erschöpfung besteht », erlaubt, nicht aus ? Welche Instanz wäre befugt, etwaige Konflikte zwischen den WTO-Regeln und den Handelsbestimmungen der MEA zu beizulegen ? Auf diese und ähnliche Fragen muss der CTE eine Antwort geben.

\section{Liste der wichtigsten von der Schweiz ratifizierten multilateralen Umweltabkommen}

- Konvention über den internationalen Handel mit vom Aussterben betroffenen Tier- und Pflanzenarten

- Übereinkommen zum Schutz des Rheins gegen Verunreinigung durch Chloride

- Übereinkommen über die Erhaltung der europäischen wildlebenden Pflanzen und Tiere und ihrer natürlichen Lebensräume

- Übereinkommen über weiträumige grenzüberschreitende Luftverunreinigung. Die Schweiz hat bereits vier der fünf Protokolle des Übereinkommens ratifiziert.

- Wiener Übereinkommen zum Schutz der Ozonschicht

- Montrealer Protokoll über Stoffe, die zu einem Abbau der Ozonschicht führen. Die Schweiz hat die beiden Änderungen des Protokolls ebenfalls ratifiziert.

- Basler Übereinkommen über die Kontrolle der grenzüberschreitenden Verbringung gefährlicher Abfälle und ihrer Entsorgung

- Konvention über Klimaänderungen

- Konvention über die Artenvielfalt

- Konvention über die Bekämpfung der Wüstenbildung in von Dürre und/oder Wüstenbildung schwer betroffenen Ländern, insbesondere in Afrika.

5 Wenn die bilateralen Abkommen dazugezählt werden, sind es über 1'000.

${ }^{6}$ Bezüglich bestimmter Handelsmassnahmen besteht allerdings die Vermutung der Unvereinbarkeit mit den WTORegeln. Das bekannteste Beispiel ist die jüngste (im September 1995) beschlossene Änderung des Basler Übereinkommens, welche den grenzüberschreitenden Transport gefährlicher Abfälle zwischen OECD-Ländern und Nichtmitgliedern der OECD verbietet. Diese Bestimmung wird oft als diskriminierend bewertet. 


\section{$\square$ Haltung verschiedener Partner}

Dieser Punkt des Arbeitsprogrammes steht eindeutig im Mittelpunkt der Diskussionen im CTE. Zahlreiche Ländern haben dazu Vorschläge eingereicht. Allerdings konnte kein Konsens erzielt werden, da die Positionen erheblich voneinander abweichen. Die meisten Länder möchten die Frage beleuchten, ob in MEA getroffene Handelsmassnahmen mit den aktuellen Bestimmungen der WTO vereinbar sind ; einige Staaten sind jedoch gegenteiliger Ansicht und ziehen den Status quo vor. Interessanterweise führen die verschiedenen Delegationen für letztere Option sehr unterschiedliche Gründe an : manche Länder legen die WTORegeln sehr strikt aus und meinen, dass dadurch der Einsatz von Handelsmassnahmen eingeschränkt werde ; andere hingegen halten die geltenden Regeln für ausreichend flexibel, um den MEA-Verhandlungspartnern gegebenenfalls den Rückgriff auf Handelsbestimmungen zu erlauben.

Auch die Auffassungen jener Delegationen, welche eine Klärung des Verhältnisses zwischen der WTO und den MEA wünschen, variieren beträchtlich. Einige befürworten eine Lockerung der Handelsbestimmungen zugunsten des Umweltrechtes, andere hingegen möchten dieses stärker einschränken. Die vorgeschlagenen Lösungen lassen sich schematisch zwei Kategorien zuordnen : ex post Ansatz und ex ante-Ansatz.

Der ex ante-Ansatz besteht darin, mit Blick auf künftige MEA-Verhandlungen die Vorhersehbarkeit bezüglich der mit den WTO-Regeln vereinbaren Handelsbeziehungen zu verbessern. Dies könnte in Form einer gemeinsamen Auslegungserklärung oder einer Änderung der allgemeinen Ausnahmen (Artikel XX des GATT) erfolgen. So würden die aufgrund von MEA getroffenen Handelsmassnahmen ausdrücklich geregelt. Diese Auffassung wird, vereinfacht dargestellt, vor allem von den OECD-Ländern vertreten.

Andere Delegationen (mehrheitlich Entwicklungsländer) plädieren für einen $e x$ post-Ansatz, wobei sie sich auf Artikel IX des WTO-Abkommens abstützen. Für Handelsmassnahmen aus einem MEA würde je nach Fall eine befreiende Bestimmung vorgesehen. Den Handelsmassnahmen bestimmter MEA könnten also befristete Befreiungen (waivers) gewährt werden.

Schliesslich steht die Frage der Beilegung etwaiger Streitigkeiten zwischen der WTO und den MEA zur Diskussion. Auch in diesem Punkt gehen die Meinungen auseinander. Manche Länder (namentlich die Entwicklungsländer) fordern, alle Streitigkeiten im Rahmen der WTO beizulegen; einige halten die WTO sogar für befugt, die Notwendigkeit und Effizienz von Massnahmen zu beurteilen, welche zur Verwirklichung von Umweltzielen getroffen werden. Nach der Meinung anderer Staaten hingegen sollten die MEA-Parteien ihre Konflikte durch Rückgriff auf den im entsprechenden Abkommen vorgesehenen Streitbeilegungsmechanismus schlichten ; Streitigkeiten zwischen Mitgliedern und Nichtmitgliedern eines MEA könnten jedoch im Rahmen der WTO ausgetragen werden.

\section{$\square$ Haltung der Schweiz}

Die Schweiz hält diesen Punkt des Arbeitsprogrammes für wesentlich und befürwortet eine Klärung der Regeln. Es ist davon auszugehen, dass noch weitere

7 Die Unterteilung der Vorschläge in zwei Kategorien bedeutet eine Vereinfachung. Es gibt keinen reinen « ex ante » Ansatz, sondern lediglich Lösungen, die ex ante- und ex post-Phasen kombinieren. 
MEA mit Handelsregeln ausgehandelt werden. Nach Ansicht der Schweiz soll eine Hierarchie zwischen der WTO und den MEA vermieden werden, die Autonomie der MEA muss gewahrt bleiben. In diesem Zusammenhang hat die Schweiz einen doppelten Ansatz vorgeschlagen, um die Frage der in Anwendung eines MEA getroffenen Handelsmassnahmen anzugehen : einerseits ex anteBestimmungen, um Konflikten vorzubeugen, andererseits ex post-Bestimmungen, um etwaige Streitigkeiten beizulegen.

Eine bessere Zusammenarbeit unter den Verantwortlichen für Handel und jenen für Umwelt auf nationaler und internationaler Ebene würde zahlreiche Konflikte vermeiden helfen. Aus diesem Grund schlägt die Schweiz vor, einen Kooperationsmechanismus zwischen der WTO und den MEA-Sekretariaten einzurichten. Der Mechanismus würde insbesondere die Gegenseitigkeit in bezug auf den Beobachterstatus und den Informationsaustausch unter den betreffenden Gremien gewährleisten.

Für den Fall, dass trotz verbesserter Abstimmung von Umwelt- und Handelspolitiken Streitigkeiten auftreten sollten, regt die Schweiz an, eine Kohärenzklausel in die WTO-Bestimmungen aufzunehmen : Bei einem Widerspruch zwischen den WTO-Regeln und einer spezifischen MEA-Handelsbestimmung würde sich die Kompetenz der WTO auf die Beurteilung beschränken, ob die Massnahme eine willkürliche Diskriminierung von Ländern mit gleichen Bedingungen darstellt, oder ob sie angewandt wird, um Handelsvorteile zu erzielen. Die WTO sollte eine Liste jener MEA erarbeiten, welche der Kohärenzklausel unterstellt sind.

\subsection{ECOLABELLING (PUNKT 3B DES CTE-ARBEITSPROGRAMMES)}

\section{$\square$ Problematik}

Die Kernfrage dieses Punktes betrifft das Verhältnis zwischen den WTO-Regeln und den Ecolabelling-Programmen. Der CTE hat insbesondere zu prüfen, ob das Übereinkommen über technische Handelshemmnisse (TBT) der WTO sich auf solche Programme anwenden lässt. Das Problem gehört in den breiteren Zusammenhang der Analyse etwaiger Auswirkungen von Ecolabelling-Programmen auf den Marktzugang, insbesondere für die Entwicklungsländer. Ferner stellt sich die heikle Frage der Produktionsverfahren und -methoden (PPM) : Zahlreiche Ecolabels stützen sich auf die Lebenszyklusanalyse der Produkte und beinhalten somit an PPM gebundene Kriterien. Sofern Produkte in ihren physischen Merkmalen durch die PPM nicht beeinflusst werden, gelten sie jedoch laut den WTORegeln als « ähnlich » und dürfen an der Grenze nicht diskriminiert werden. ${ }^{8}$

\section{$\square$ Haltung verschiedener Partner}

Die Entwicklungsländer werfen den Ecolabelling-Programmen protektionistische Auswirkungen auf den Markzugang vor. Aus diesem Grund fordern sie grössere Transparenz und ein verbessertes Konsultationsverfahren bei der Gestaltung der Programme. Allerdings zeigen diese Länder wenig Neigung, sol-

\footnotetext{
8 Das bekannteste Beispiel von PPM ohne Auswirkungen auf die Merkmale des Endproduktes ist das « Thunfisch »Panel. Aufgrund der WTO-Regeln ist die Unterscheidung an der Grenze zwischen Thunfisch, bei dessen Fang keine Delphine getötet werden, und solchem, der ohne Rücksicht auf Delphine gefischt wird, nicht zulässig.
} 
che Programme dem TBT-Übereinkommen zu unterstellen, zumal sie auf PPM gegründete Labels für mit den Bestimmungen dieses Übereinkommens und mit anderen GATT-Regeln unvereinbar halten.

Die OECD-Länder stimmen weitgehend in der Feststellung überein, dass die Transparenz der Programme erhöht werden muss ; sie sind sich jedoch uneinig über die dazu einzusetzenden Mittel. Einige plädieren für die Einführung eines Verhaltenskodexes, andere möchten die Ecolabels teilweise vom TBT-Übereinkommen abgedeckt sehen. ${ }^{9}$

\section{$\square$ Haltung der Schweiz}

Die Schweiz ist zunächst der Ansicht, dass freiwillige Etikettierungsprogramme effiziente, nach dem Mechanismus von Angebot und Nachfrage funktionierende Instrumente darstellen. Nach Einschätzung der Schweiz haben diese Instrumente gegenwärtig keine grossen Auswirkungen auf den Marktzugang. Allerdings sind mit der zunehmenden Entwicklung der Programme echte Schwierigkeiten abzusehen. Daher tritt die Schweiz für eine grössere Transparenz bei der Entwicklung der Labels ein. Wichtig erscheint ferner, dass die Labels nicht willkürlich vergeben werden. Produkte eines ausländischen Exporteurs, die den Kriterien eines nationalen Labels genügen, müssen dieses erhalten können. Schliesslich plädiert die Schweiz für horizontale Lösungen im Rahmen der WTO, um eine identische Behandlung sämtlicher Label (Sozial-, Eco-, Informatiklabel) zu gewährleisten.

Die Schweiz hat zur Frage der Anwendung des TBT-Übereinkommens auf die Labels bislang noch keine Stellung bezogen. Im übrigen ist nach Ansicht der Schweiz eine Entscheidung zu den PPM ohne Auswirkungen auf das Endprodukt verfrüht. Diese Frage sollte in einem umfassenderen Rahmen als in der derzeit laufenden Diskussion zum Ecolabelling geprüft werden.

\subsection{EXPORTE VON AUF DEM INLÄNDISCHEN MARKT VERBOTENEN PRODUKTEN (PUNKT 7 DES CTE- ARBEITSPROGRAMMES)}

\section{$\square$ Problematik}

Hier geht es um Produkte, deren Verkauf und Verwendung auf dem inländischen Markt wegen der Gefahr für die Gesundheit und das Leben von Menschen und Tieren oder aus Gründen des Pflanzenschutzes eingeschränkt oder verboten sind, deren Export jedoch gestattet ist. Bereits 1991 hatte sich eine Arbeitsgruppe mit der Frage auseinandergesetzt. Sie erarbeitete einen Beschlussentwurf über Produkte, die auf dem inländischen Markt verboten oder streng reglementiert sind. Mangels eines Konsenses wurde der Beschluss jedoch nie verabschiedet. Seitdem sind mehrere neue Abkommen und Konventionen zu diesem Thema bereits in Kraft getreten oder werden unter der Ägide anderer internationaler Organisationen (UNEP, FAO, IAA usw.) noch ausgehandelt. Der CTE hat nun die Aufgabe, die mögliche Rolle der WTO in diesem Bereich zu definieren.

9 Das TBT-Übereinkommen würde allerdings nur obligatorische und freiwillige Labels, ohne Bezug zu auf die Lebenszyklusanalyse abgestützten Kriterien, betreffen. Die Frage der PPM bliebe somit offen. 


\section{$\square$ Haltung verschiedener Partner.}

Dieses Thema bereitet vielen Entwicklungsländern Sorgen. Sie hatten darauf bestanden, den Punkt in das Arbeitsprogramm des CTE aufzunehmen. Nach Ansicht der Entwicklungsländer kann die WTO nämlich auf sinnvolle Weise zur Erhöhung der Transparenz im Handel mit solchen Produkten beitragen. Eine Delegation unterbreitete dem CTE sogar einen neuen Beschlussentwurf, der die einschlägigen internationalen Abkommen - insbesondere hinsichtlich des Geltungsbereichs - ergänzen soll. So wird vorgeschlagen, dass der Beschluss Produkte wie Lebensmittelzusätze, Konsumgüter, Abfälle, Kosmetika und Arzneimittel erfassen soll. Der Beschluss würde die Exportländer verpflichten, die von ihnen ausgeführten verbotenen Produkte den anderen WTO-Mitgliedern zu notifizieren. Ferner werden Bestimmungen zu Massnahmen über die technische Unterstützung in diesem Bereich sowie die Schaffung von Informationsstellen vorgeschlagen. Schliesslich werden die Exportländer aufgefordert, zu überprüfen, ob die im Inland für die fraglichen Produkte angewandten Massnahmen nicht auch für ihre Ausfuhren gelten sollen.

\section{$\square$ Haltung der Schweiz}

Die Schweiz ist generell an dieser Problematik interessiert. Sie beteiligt sich aktiv an den in anderen internationalen Foren laufenden Verhandlungen (in den Bereichen Chemiegüter, Pestizide und Abfälle). Die Schweiz betont, dass in der WTO Doppelarbeiten zu den Bemühungen anderer Organisationen zu vermeiden sind. Das Vorgehen der WTO in diesem Bereich muss subsidiär bleiben und soll sich auf jene Produkte beschränken, die von keinem anderen internationalen Abkommen erfasst werden.

Daneben äusserte die Schweiz Zweifel an der Befugnis des CTE, sich mit der technischen Unterstützung zu befassen. Die Schweiz vertritt den Standpunkt, dass die WTO nicht über das erforderliche Fachwissen auf diesem Gebiet verfügt und dass eine Sonderorganisation diese Aufgabe effizienter wahrnehmen könnte.

\subsection{ABKOMMEN ÜBER DAS GEISTIGE EIGENTUM (PUNKT 8 DES CTE-ARBEITS- PROGRAMMS)}

\section{- Problematik}

Der CTE erhält die Aufgabe, das Abkommen über die handelsbezogenen Aspekte der Rechte an geistigem Eigentum (TRIPS) im Zusammenhang mit folgenden Fragen zu überprüfen: Umwelt, Entwicklung ökologisch rationeller Technologien, Zugang zu und Transfer solcher Technologien, traditionelle Kenntnisse der autochthonen Gemeinschaften.

\section{$\square$ Haltung verschiedener Partner}

Zu diesem Punkt des Arbeitsprogrammes vertreten die Industrie- und Entwicklungsländer ausgeprägte und sehr unterschiedliche Meinungen. Die Entwicklungsländer sehen in den geistigen Eigentumsrechten ein Hindernis für den Transfer umweltfreundlicher Technologien. Daher ist ihrer Ansicht nach zu überprüfen, ob gewisse Bestimmungen des TRIPS-Abkommens die Anschaffung der erforderlichen Technologien in den Entwicklungsländern behindern könnten. Manche Ländern vertreten die Auffassung, dass die Rechte an geistigem Eigen- 
tum die Technologien verteuern. Ferner stellen bestimmte Entwicklungsländer Forderungen hinsichtlich der traditionellen Kenntnisse der autochthonen Gemeinschaften, welchen das TRIPS-Abkommen nicht Rechnung trage. Sie halten die Verabschiedung neuer Gesetze für notwendig, damit die Träger der traditionellen Kenntnisse Vorteile aus deren Nutzung durch ausländische Unternehmen erlangen. Untermauert wird diese Forderung durch eine Bestimmung der Konvention über die Artenvielfalt, welche vorsieht, dass jede Vertragspartei die gerechte Teilung der aus der Nutzung dieser Kenntnisse, Innovationen und Gebräuche der eingeborenen und ortsansässigen Gemeinschaften fördern soll.

Nach Auffassung der meisten Industrieländer gehören traditionelle Kenntnisse der autochthonen Bevölkerung nicht zum geistigen Eigentum, zumal für weit verbreitete oder zu Gemeingut gewordene Kenntnisse keine Rechte an geistigem Eigentum gewährt werden können. Freiwillige Abkommen zwischen Unternehmen, Regierungen und autochthoner Bevölkerung stellen die einzige effiziente Methode dar, um traditionelle Kenntnisse zu « bezahlen ».

\section{$\square$ Haltung der Schweiz}

In den Augen der Schweiz stellt das System zum Schutz der geistigen Eigentumsrechte einen Anreiz für Innovationen und mithin für die Entwicklung umweltfreundlicher Technologien dar. Zudem glaubt die Schweiz, dass das System auch den Technologietransfer fördert. Andere Faktoren, wie die wirtschaftliche und politische Stabilität des Empfängerlandes, Infrastrukturen, Zugang zu Finanzressourcen und Berufsausbildungsstand fallen jedoch erheblich stärker ins Gewicht. Schliesslich hält die Schweiz es für verfrüht, Änderungen des TRIPS-Abkommens zu erwägen, solange dieses noch nicht vollständig anwendbar ist (die Entwicklungsländern verfügen über eine Übergangsfrist).

\subsection{AUSWERTUNG DER ARBEITEN DES CTE}

Die vom CTE durchgeführten Arbeiten zeigten die Hauptprobleme auf, vor denen die Entwicklungsländer stehen, und verwiesen auf Fragen, die noch gelöst werden müssen, um Umwelt- und Handelspolitik besser aufeinander abzustimmen. Von der WTO sollte man sich allerdings nicht zuviel versprechen. Ihr Mandat besteht in der Tat darin, den Freihandel zwischen den Staaten voranzutreiben, und nicht Gesetze im Umweltbereich zu schaffen. Hinzu kommt, dass zahlreiche Delegationen im CTE nur aus Handelsexperten bestehen, welche weder mit der Umweltproblematik noch mit den in anderen Foren laufenden Diskussionen vertraut sind. So kann es geschehen, dass ein Delegierter eines bestimmten Landes im CTE für das Verbot von Handelsmassnahmen plädiert, die im Rahmen eines MEA getroffen wurden, und dass dasselbe Land kurz zuvor die Einführung dieser Massnahmen in eine Umweltkonvention gefordert hatte.

Angesichts der abweichenden Meinungen in den meisten Punkten des CTEArbeitsprogrammes sind die Erwartungen an mögliche Ergebnisse in den nächsten Jahren relativ pessimistisch. Der allgemeinen Ansicht zufolge wäre ein Erfolg der aktuellen Verhandlungen in der WTO nur möglich, wenn ein ganzes Paket an Entscheidungen zu verschiedenen Punkten des Arbeitsprogrammes ausgehandelt würde. Sektorielle - d.h. nur einen Punkt des Arbeitsprogrammes betreffende - Verhandlungen hätten geringe Chancen, ein für alle WTO-Mitglie- 
der zufriedenstellendes Ergebnis zu erzielen. Gegenwärtig zeichnet sich jedoch noch keine « Globallösung » ab ; die Delegationen beharren auf ihren Positionen und verweigern Zugeständnisse. Aus diesem Grund ist das Mandat des CTE zu verlängern, damit die Verhandlungen zu sämtlichen Punkten des Arbeitsprogrammes fortgesetzt werden können.

\section{STAND DER DISKUSSIONEN IN ANDEREN INTERNATIONALEN ORGANISATIONEN}

\subsection{KONFERENZ DER VEREINTEN NATIONEN FÜR HANDEL UND ENTWICKLUNG (UNCTAD)}

Die Sonderarbeitsgruppe zu Handel, Umwelt und Entwicklung wurde vom Rat für Handel und Entwicklung der UNCTAD eingesetzt. Sie traf 1995 mehrmals zusammen, um

- die Auswirkungen von Politiken, Normen und Vorschriften im Umweltbereich auf den Marktzugang und auf die Wettbewerbsfähigkeit zu überprüfen ;

- neue umweltpolitische Instrumente, die den Handel beeinflussen, auszuarbeiten und zu analysieren ;

- Ecolabelling- und Ecozertifizierungs-Programme zu studieren und die Möglichkeiten einer internationalen Zusammenarbeit in diesem Bereich zu prüfen.

In den Diskussionen der Gruppe zeichnete sich in allen behandelten Punkten eine tiefgehende Spaltung zwischen den Entwicklungs- und den Industrieländern ab. Die Entwicklungsländer beharrten ziemlich unnachgiebig auf ihrem Standpunkt und warfen den Umweltpolitiken der Industrieländer eine erhebliche Beeinträchtigung ihres eigenen Marktzugangs und ihrer Wettbewerbsfähigkeit vor (insbesondere in den Sektoren Textilien, Leder und Schuhe). "Einige Delegationen äusserten die Befürchtung, dass die Gewinne aus der Uruguay-Runde durch die immer strengeren ökologischen Auflagen aufgehoben werden könnten » (Übersetzung aus dem französischen Originaltext). ${ }^{10}$

Die Auffassung der Industrieländer zu den Auswirkungen der Umweltpolitiken auf die Wettbewerbsfähigkeit lautet sehr unterschiedlich. So erinnerten die anwesenden Delegationen an den Inhalt des Rapport sur les échanges et l'environnement au Conseil au niveau des Ministres (OECD, 1995), dessen Hauptergebnisse unter Punkt 3.2 unten zusammengefasst sind.

Nach heftigen Auseinandersetzungen gelang es schliesslich, einige Empfehlungen zu unterbreiten. Darin werden die Regierungen angesichts der « besonderen Situation der Entwicklungsländer ${ }^{11}$ vor allem aufgefordert, positive Massnahmen zu ergreifen, z.B. mit der Verbesserung des Markzutritts, des Zugangs zu Finanzressourcen und zu Technologie. Ferner wiederholen die Empfehlungen bestimmte Prinzipien wie die Verurteilung unilateraler, extraterritorialer Massnahmen zur Lösung von Umweltproblemen, verbesserte Transparenz von Umweltmassnahmen (insbesondere in bezug auf das Ecolabelling) und die gemeinsame, wenngleich differenzierte Verantwortung bei der Bewältigung von Umweltproblemen.

\footnotetext{
${ }^{10}$ Schlussbericht der Sondergruppe, S. 8.

${ }^{11}$ Ibid., S. 12.
} 


\subsection{ORGANISATION FÜR WIRTSCHAFTLICHE ZUSAMMENARBEIT UND ENTWICKLUNG (OECD)}

Die im Frühjahr 1991 eingeführte gemeinsame Tagung der Handels- und Umweltsachverständigen stellt das einzige Forum dar, in welchem Experten aus dem Handels- und Umweltbereich paritätisch vertreten sind. Bis heute hat die Arbeitsgruppe einige Berichte erarbeitet, darunter der letzte, vom Mai 1995 datierte Bericht mit dem Titel «Le rapport sur les échanges et l'environnement au Conseil au niveau des Ministres ». Der Bericht enthält eine Reihe von Schlussfolgerungen und Empfehlungen. Er kann zwar nicht als revolutionär bezeichnet werden, stellt aber eine erste Etappe des Reflexionsprozesses dar. So erlaubte er den OECD-Ländern, Bilanz zu ziehen und zu einigen wichtigen Themen eine gemeinsame Position zu erarbeiten. Die wichtigsten Schlussfolgerungen des Berichts werden hier zusammengefasst dargestellt :

\section{Auswirkungen der Handelsliberalisierung auf die Umwelt}

Die Folgen der Handelsliberalisierung auf die Umwelt sind noch sehr umstritten. Da keine echte Einigung zu diesen Auswirkungen zustande kam, stellten die OECD-Länder lediglich fest, dass « die Handelsliberalisierung sich im allgemeinen günstig auf die Umwelt auswirkt, indem sie eine effizientere Ressourcenverteilung ermöglicht, das Wirtschaftswachstum fördert und die allgemeine Wohlfahrt steigert, unter der Voraussetzung, dass wirksame Umweltpolitiken umgesetzt werden ${ }^{12}$

\section{$\square$ Fragen zur Wettbewerbsfähigkeit}

Gemäss den OECD-Ländern kann ein hohes Umweltschutzniveau sich positiv auf die Wettbewerbsfähigkeit der nationalen Produzenten und der Länder auswirken. «Ein hohes Umweltschutzniveau kann die technologischen Fortschritte ankurbeln, Investitionen fördern, die Effizienz der Produktion steigern und gleichzeitig die Entwicklung neuer Industriesektoren und neuer Absatzmärkte bewirken $»^{13}$ (Übersetzung aus dem französischen Originaltext). Zur befürchteten Einbusse an Wettbewerbsfähigkeit unterstreichen die Experten, dass anhand der bislang durchgeführten Arbeiten keine systematische Beziehung zwischen geltenden Umweltpolitiken und Auswirkungen auf die Produktivität nachgewiesen werden konnte.

\section{$\square$ Produktionsverfahren und -methoden (PPM)}

Die OECD-Länder betonen, dass Befürchtungen bezüglich der grenzüberschreitenden, globalen Auswirkungen der PPM im Rahmen der internationalen Zusammenarbeit angegangen werden sollen. Die Zusammenarbeit kann in der Form von technischer und finanzieller Unterstützung, durch die Aushandlung multilateraler Umweltabkommen oder durch die internationale Normenharmonisierung stattfinden.

\footnotetext{
${ }^{12}$ Rapport sur les échanges et l'environnement au Conseil au niveau des Ministres, S. 4.

${ }^{13}$ Ibid., S. 4.
} 


\section{SCHLUSSFOLGERUNGEN}

Die Analyse der Beziehung von Handel und Umwelt ist in den letzten Jahren erheblich vorangekommen. Mehrere Organisationen setzten Sachverständigengruppen zu diesem Thema ein ; daneben wurden zahlreiche empirische Studien durchgeführt. Trotzdem führt das Thema weiterhin zu Spannungen zwischen Umwelt- und Handelsexperten wie auch zwischen Industrie- und Entwicklungsländern. Die Arbeiten des Ausschusses für Handel und Umwelt der WTO stecken gegenwärtig in einer Sackgasse. Dies zeigt, dass erhebliche Anstrengungen erforderlich sind, um sämtliche Standpunkte in Einklang zu bringen und für alle akzeptierbare Lösungen zu finden.

Wichtig ist in diesem Zusammenhang, dass die Länder in den verschiedenen internationalen Organisationen (namentlich im Rahmen des UNEP und der WTO) ihre Positionen auf kohärentere Weise vertreten. Zudem sollten vermehrt Umweltexperten an den Arbeiten des CTE beteiligt werden.

Auch die Rolle des UNEP in dieser Diskussion ist neu zu definieren und auszubauen. Das UNEP sollte mit den notwendigen Mitteln ausgestattet werden, um einige rechtliche Bestimmungen der MEA, die unter seine Verantwortung fallen, zu verbessern und so die Stellung dieser Abkommen gegenüber der WTO zu festigen (insbesondere hinsichtlich des Streitbeilegungsmechanismus).

Laut Auffassung der Schweiz ist eine Übereinstimmung der bislang geäusserten unterschiedlichen Meinungen durchaus möglich. In der Tat bedarf es einer Ergänzung von Handels- und Umweltpolitiken, um die nachhaltige Entwicklung des Planeten zu gewährleisten. Die Ängste mancher Entwicklungsländer vor einem « grünem Protektionismus » müssen ernst genommen werden. Die Industrieländer sollten ihren Partnern Garantien zur Transparenz geben, um so die Dauerhaftigkeit und die Festigung eines gerechten und offenen multilateralen Handelssystems zu sichern.

QUELLEN

Dirk von Felbert, Echanges, environnement et aide, in L'Observateur de l'OCDE, Nr. 195, August-September 1995, S. 610.

Trade \& the Environment : the Search for Balance, Edited by James Cameron, Paul Demaret \& Damien Geradin, Cameron May Ltd., 1994.

James Cameron, Halina Ward, The Multilateral Trade Organisation, A Revised Prospective, Paper presented for the International Conference « striking a green deal », Brussels, 7-9 November 1993.

Rapport final du Groupe de travail spécifique sur le commerce, l'environnement et le développement, UNCTAD, Genf, November 1995.

Rapport sur les échanges et l'environnement au Conseil au niveau des Ministres, OECD, Paris, Mai 1995.

Résultats des négociations commerciales multilatérales du Cycle d'Uruguay, Rechtstexte, GATT-Sekretariat, Mai 1996.

Commerce et environnement, Nouvelles et points de vue de l'OMC (Pressecommuniqué), Nr. 1-11. 